INPLASY

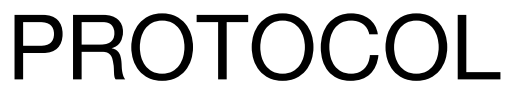

To cite: Lin et al. A systematic review and Meta-analysis of effectiveness of acupuncturing Trigger Points for treatment of Myofascial Pain Syndrome. Inplasy protocol 202120073. doi:

10.37766/inplasy2021.2.0073

Received: 20 February 2021

Published: 21 February 2021

Corresponding author:

Sha Yang

303300670@qq.com

Author Affiliation:

School of acupuncture and moxibustion and tuina, Chengdu University of Traditional Chinese Medicine, P.R.China.

Support: Chengdu University of TCM.

Review Stage at time of this submission: Formal screening of search results against eligibility criteria.

\section{A systematic review and Meta- analysis of effectiveness of acupuncturing Trigger Points for treatment of Myofascial Pain Syndrome}

Lin, D1; Fu, Q2; Liu, Y3; Chen, X4; Lei, H5; Yang, S6.

Review question / Objective: For patients with myofascial pain syndrome, to explore whether the trigger points of conventional acupuncture needles are better than other therapies in terms of visual analog scale (VAS), clinical efficacy, present pain intensity (PPI) and pain rating index (PRI) effective?

Condition being studied: Myofascial pain syndrome (MPS) is a chronic pain symptom caused by aseptic inflammation of muscles and fascia and the formation of trigger points (MTrP). MPS belongs to the category of TCM Jinbi. Mainly manifested as local pain, chills, numbness, muscle cramps and movement disorders. Pain is common and usually has obvious trigger points. Touching this point may cause radiating pain. The incidence of MPS is relatively high. After an upper respiratory tract infection.

INPLASY registration number: This protocol was registered with the International Platform of Registered Systematic Review and Meta-Analysis Protocols (INPLASY) on 21 February 2021 and was last updated on 21 February 2021 (registration number INPLASY202120073).

Conflicts of interest:

None declared.

\section{INTRODUCTION}

Review question / Objective: For patients with myofascial pain syndrome, to explore whether the trigger points of conventional acupuncture needles are better than other therapies in terms of visual analog scale (VAS), clinical efficacy, present pain intensity (PPI) and pain rating index (PRI) effective? 
Condition being studied: Myofascial pain syndrome (MPS) is a chronic pain symptom caused by aseptic inflammation of muscles and fascia and the formation of trigger points (MTrP). MPS belongs to the category of TCM Jinbi. Mainly manifested as local pain, chills, numbness, muscle cramps and movement disorders. Pain is common and usually has obvious trigger points. Touching this point may cause radiating pain. The incidence of MPS is relatively high. After an upper respiratory tract infection.

\section{METHODS}

Participant or population: Inclusion criteria: Main standards: Complaints of regional pain; Complaints of pain or paraesthesia where the trigger point involves the expected distribution area of pain; The affected muscles can touch the tension zone; A certain point in the tension zone is intensely tender; At the time of measurement, there is a certain degree of movement restriction. Additional criteria: The main complaint of clinical pain or paresthesia recurring at the tender point; Lateral scratching or acupuncture at the trigger point of the human band to induce local twitch response; Stretch muscles or inject trigger points to relieve pain. MPS can be diagnosed only if 5 major and at least 1 minor criteria are met. The study populations were not restricted by the location of MPS, age, gender and race. Exclusion criteria: Non-randomized controlled trial research literature; Documents published repeatedly (same data); Combination of treatment and the use of other acupuncture therapies, other treatment methods, Chinese medicine, Chinese patent medicine, Western medicine or other surgical treatment methods; Outcome indicators do not meet the inclusion criteria or data cannot be obtained; Case/control (comparison of disease group and non-disease group) study.

Intervention: Conventional Acupuncture Needles to Acupuncture Trigger Point for Treatment of Myofascial Pain Syndrome.
Comparator: The treatment group was given conventional acupuncture needle trigger points; the control group was given other therapies besides conventional acupuncture needles, such as lidocaine b l ock therapy, low-frequency transcutaneous electrical stimulation therapy, extracorporeal shock wave therapy, etc.

Study designs to be included: Randomized controlled trials, no blinding restrictions. Refer to Article 12 for inclusion and exclusion criteria.

Eligibility criteria: Inclusion criteria: Main standards: Complaints of regional pain; Complaints of pain or paraesthesia where the trigger point involves the expected distribution area of pain; The affected muscles can touch the tension zone; A certain point in the tension zone is intensely tender; At the time of measurement, there is a certain degree of movement restriction. Additional criteria: The main complaint of clinical pain or paresthesia recurring at the tender point; Lateral scratching or acupuncture at the trigger point of the human band to induce local twitch response; Stretch muscles or inject trigger points to relieve pain. MPS can be diagnosed only if 5 major and at least 1 minor criteria are met. The study populations were not restricted by the location of MPS, age, gender and race. Exclusion criteria: Non-randomized controlled trial research literature; Documents published repeatedly (same data); Combination of treatment and the use of other acupuncture therapies, other treatment methods, Chinese medicine, Chinese patent medicine, Western medicine or other surgical treatment methods; Outcome indicators do not meet the inclusion criteria or data cannot be obtained; Case/control (comparison of disease group and non-disease group) study.

Information sources: Articles will be searched from January 2021 to February 2021. Includes English and Chinese articles. The literature search will be 
conducted in the following databases: PubMed, Cochrane library, EMBASE, MEDLINE, Web of science, Wanfang, CNKI, CBM, VIP. The search strategy will use the description of acupuncture trigger points as an intervention for patients with myofascial pain syndrome.

Main outcome(s): Visual analogus scale (VAS); Clinical efficacy. This is measured pre-intervention and post-intervention.

Additional outcome(s): Present pain intensity (PPI); Pain rating index (PRI). This is measured pre-intervention and postintervention.

Quality assessment / Risk of bias analysis: The RCT bias risk evaluation criteria in the Cochrane Collaboration were used to evaluate the methodological quality of a single RCT. The quality evaluation is carried out by two researchers independently and reviewed with each other. In case of differences, they will be discussed or decided with reference to the opinions of a third party.

Strategy of data synthesis: The total participant data will be used to develop a combination of quantitative and qualitative. Our main outcomes measures the visual analogus scale (VAS) and clinical efficacy. The additional outcomes measures the present pain intensity (PPI) and the pain rating index (PRI). Four meta-analysis will be conducted: VAS, clinical efficacy, PPI, PRI. The data will be analyzed using Revman5.3 software. Two researchers will participate in this process. The discrepancy will be resolved by involving a third examiner.

Subgroup analysis: According to the different intervention methods of the control group, they were divided into three groups: lidocaine block group, lowfrequency electrical stimulation group, and physical therapy group. Then, according to the outcome indicators: VAS, clinical efficacy, PPI, PRI for qualitative or quantitative synthesis.
Sensitivity analysis: After excluding the research with low methodological quality, analyze again and compare the analysis results before and after. If the outcome indicators are in the same direction, it means that the analysis results are stable and the conclusions drawn are reliable; otherwise, the analysis results are unstable and the results The conclusion is not reliable. At the same time, it is possible to compare whether the comprehensive results under the fixed and random effect models are different. If the results of the fixed effect model cannot reverse the results of the random effect model, the conclusion is reliable.

Language: English.

\section{Country(ies) involved: China.}

Keywords: Acupuncture Therapy; Trigger Points; Myofascial Pain Syndromes; Randomized Controlled Trial; systematic review; Meta-analysis.

Contributions of each author:

Author 1 - Dezhi Lin - Coordinating the work of the team, countermeasure research and the drafting of the paper; training of evaluation norms for members of the research group; project planning, application and demonstration; project summary information and data analysis.

Email: 627796362@qq.com

Author 2 - Qinwei Fu - Guide the design of related research methods, research indicators, research routes and other aspects of the research group; accept group academic consultation.

Email: 416650376@qq.com

Author 3 - Yi Liu - Subject summary information, data analysis; subject related survey and literature review.

Email: 2176285261@qq.com

Author 4 - Xinyi Chen - Subject summary information, data analysis; computer technology processing.

Email: 1311750632@qq.com

Author 5 - Hang Lei - Subject related surveys and literature review; subject writing bids, survey reports and papers.

Email: 812146695@qq.com 
Author 6 - Sha Yang - Guide the design of related research methods, research indicators, research routes and other aspects of the research group; accept group academic consultation.

Email: 303300670@qq.com 